\title{
Effects of GPR81 silencing combined with cisplatin stimulation on biological function in hypopharyngeal squamous cell carcinoma
}

\author{
QIAOJING JIA, OU XU, JIANXING WANG, JINHUI DONG, \\ XIUMIN REN, XIAOFANG JIA and CHUNGUANG SHAN
}

\begin{abstract}
E.N.T. Department, The Second Hospital of Hebei Medical University, Shijiazhuang, Hebei 050000, P.R. China
\end{abstract}
Received June 14, 2019; Accepted April 15, 2020

DOI: $10.3892 / \mathrm{mmr} .2020 .11255$

\begin{abstract}
Hypopharyngeal squamous cell carcinoma (HSCC) is a malignant tumor found in the head and neck region. Lactate receptor 1, also known as $\mathrm{G}$ protein-coupled receptor81 (GPR81), has been reported to play a vital role in cancer growth and metabolism. However, the function of GPR81 in HSCC remains largely unknown. The present study investigated the effect of GPR81 on cell survival and GPR81-mediated energy metabolism under cisplatin treatment in HSCC. GPR81 knockdown reduced the proliferation and invasion of the human HSCC cell line FaDu. Furthermore, GPR81 silencing combined with cisplatin treatment increased the expression of translocase of outer mitochondrial membrane 20 at the mRNA and protein levels $(\mathrm{P}<0.05)$. mRNA and protein expression of phosphofructokinase 1 in mRNA appeared to be downregulated in GPR81 knockdown FaDu cells treated with cisplatin, although this was not statistically significant. GPR81 silencing and cisplatin challenge showed no significant upregulation compared with the control, but significant downregulation in mRNA and protein levels compared with the shRNA-scramble group. Apoptosis was measured by flow cytometry with annexin $\mathrm{V}$ and 7-aminoactinomycin D. GPR81 silencing and cisplatin led to an increased apoptotic rate. Moreover, absence of GPR81 combined with cisplatin exposure increased caspase-3 expression and decreased Bcl-2 levels. The results of the present study suggested that GPR81 and cisplatin sensitivity played an important role in HSCC growth and metabolism.
\end{abstract}

\section{Introduction}

Head and neck cancer is historically common and lethal in adults worldwide (1). As a frequently occurring malignancy,

Correspondence to: Professor Chunguang Shan, E.N.T. Department, The Second Hospital of Hebei Medical University, 215 Hepingxi Road, Shijiazhuang, Hebei 050000, P.R. China E-mail: shanchunguang@163.com

Key words: G protein-couple receptor 81, silencing, cisplatin, biological function, hypopharyngeal squamous cell carcinoma hypopharyngeal squamous cell carcinoma (HSCC) is a major focus of research due to its high morbidity and mortality rates. The incidence of HSCC, regardless of age and sex, has increased, particularly in the developing world (2). Risk factors for HSCC include alcohol use, tobacco exposure, betel quid chewing, HPV infection and personal history, such as genetics, toxin exposure, diet and environmental factors $(3,4)$. Due to advances in diagnostic and therapeutic modalities, the survival rate for hypopharyngeal cancer has improved since 1990 (5). However, $\sim 3,000$ new cases are diagnosed annually in the USA (6). Thus, a better understanding of the molecular mechanisms underlying HSCC progression will likely provide insight regarding the treatment of HSCC on an individual basis.

Tumors have large bio-energetic requirements for the maintenance of cell growth and proliferation. The hypoxic and glucose-poor tumor micro-environment requires tumor cells to alter their metabolic patterns to adapt to these conditions (7). A high rate of glycolysis, with production of lactate and decreasing mitochondrial oxidative phosphorylation (OXPHOS) metabolism is a universal phenomenon in carcinoma cells $(8,9)$, which results in the accumulation of lactate within cells. Lactate in the tumor micro-environment is catalyzed by lactate dehydrogenase to synthesize pyruvate, and provide metabolic fuel for OXPHOS.

Lactate also takes part in the production of ATP, as part of the tricarboxylic acid cycle $(10,11)$, and can also be used as a substrate for gluconeogenesis (12). In the process of energy metabolism, the accumulation of lactate tends to rely on the cell membrane receptor G-protein coupled receptor 81 (GPR81) and monocarboxylase transporters (MCTs). GPR81 is mainly expressed in adipocytes, skeletal muscle and brain, and is involved in energy metabolism (13-15). Previously, GPR81 was observed to be important in various types of cancer, participating in cell survival, metastasis, tumor growth and chemoresistance (16-18). GPR81 is activated by lactate, which inhibits the conversion of ATP to cAMP (19). MCT1 has a high affinity binding ability for lactate and is mainly responsible for lactate uptake $(20,21)$. Roland et al (18) demonstrated that GPR81 is important for cancer cell regulation of lactate transport mechanisms, and alters the expression of MCT1 and MCT4 in the presence of lactate and glucose.

Phosphofructokinase 1 (PFK-1) is a primary control enzyme in the glycolytic pathway, which catalyzes the phosphorylation 
of fructose 6-phosphate to fructose 1,6-bisphosphate, accompanied by ATP conversion to ADP. In tumors, PFK-1 levels are increased compared with non-tumor cells $(18,22)$, suggesting that PFK-1 might be a functional biomarker of abnormal energy metabolism. As a key rate-limiting enzyme, the modification and alteration of PFK-1 in glycolysis can disturb the glycolytic pathway and result in metabolic disorders.

Tumor cells consume glucose through anaerobic glycolysis and generate lactate and ATP even in the presence of oxygen, which is known as the Warburg effect (23). Glycolysis and OXPHOS co-exist in cancer cells and facilitate tumorigenesis and metastasis (24).

Translocase of the outer mitochondrial membrane 20 (TOMM20) is a key subunit of the TOM complex and a vital mitochondrial transport protein. TOMM20 is regarded as a positive marker of OXPHOS (25) and is associated with many malignant tumors $(25,26)$.

Lactate, which is mainly generated by glycolysis in tumors, was recently identified as a major fuel for OXPHOS and an activator of energy conversion signaling pathways (27). To the best of the authors' knowledge, only a few previous publications have studied the effect of GPR81 silencing and cisplatin treatment on cell survival and energy metabolism in HSCC. Therefore, in the present study, several factors associated with glycolysis and OSPHOS were analyzed. The molecular role of GPR81 in the HSCC cell line FaDu was investigated. Furthermore, the influence of silencing GPR81 combined with cisplatin on the expression of PFK-1 and TOMM20 was studied, in order to identify the role played by GPR81 in glycolysis and OXPHOS, in the context of HSCC. The effect of GPR81 knockdown combined with cisplatin treatment on cell survival was also examined.

\section{Materials and methods}

Cell lines and cell culture. The human FaDu cell line is derived from hypopharyngeal carcinoma. FaDu cells obtained from China Center For Type Culture Collection were cultured in RPMI-1640 (Gibco, Thermo Fisher Scientific, Inc.) supplemented with 10\% FBS (Hyclone; GE Healthcare Life Sciences), $100 \mathrm{U} / \mathrm{ml}$ penicillin, $100 \mathrm{mg} / \mathrm{ml}$ streptomycin and $0.1 \mathrm{M}$ HEPES in a humidified atmosphere containing $5 \% \mathrm{CO}_{2}$ at $37^{\circ} \mathrm{C}$.

Plasmid construction. The interference plasmids containing human short hairpin shRNA (shRNA)-GPR81 (also known as hHCAR1) and shRNA-scramble were obtained from Cyagen Biosciences, Inc. The shRNA-GPR81 plasmid effectively inhibited the expression of GPR81, and the shRNA-scramble plasmid acted as a control.

GPR81 knockdown and cell challenge. In order to inhibit the expression of GPR81, FaDu cells were transfected with $2.5 \mu \mathrm{g}$ shRNA-GPR81 plasmid and $2.5 \mu \mathrm{g}$ shRNA-scramble plasmid was used as a control. Transfections were carried out using Lipofectamine ${ }^{\circledR} 3000$ reagent (Invitrogen; Thermo Fisher Scientific, Inc.) according to the manufacturer's protocol. Transfection efficiency was determined in the experimental group and the control group by western blot analysis. Following transfection for $48 \mathrm{~h}$, the transfected cells were then challenged for $24 \mathrm{~h}$ with cisplatin at concentrations of $0,0.5,1$, 2 and $5 \mu \mathrm{g} / \mathrm{ml}$. Each group was plated in triplicate. Cells were collected for RNA and protein extraction.

Cell proliferation assay. Following transfection with shRNA-GPR81 and shRNA-scramble plasmid for $48 \mathrm{~h}$, cells were seeded into a 96 -well plate at a density of $5 \times 10^{3}$ cells/well and stimulated with $5 \mu \mathrm{g} / \mathrm{ml}$ cisplatin. Cell proliferation was evaluated using Cell Counting Kit-8 reagent (CCK-8; Beyotime Institute of Biotechnology) according to the manufacturer's protocol. The proliferation of $\mathrm{FaDu}$ cells was measured by the absorbance at a wavelength of $450 \mathrm{~nm}$ (OD450; where OD indicates optical density).

Transwell Matrigel ${ }^{T M}$ invasion assay. The invasive capacity of FaDu cells transfected with shRNA-GPR81 was assessed with Transwell Matrigel invasion assays. A 24-well chamber covering Matrige ${ }^{\mathrm{TM}}$-coated (diluted 1:3 in serum-free medium on ice; $\left.100 \mu 1 / \mathrm{cm}^{2}\right)$ membranes with a pore size of $8 \mu \mathrm{m}$ (Corning, Inc.) was used to assess cell invasion. Transfected cells were diluted in serum-free medium to a concentration of $1 \times 10^{5}$ cells $/ \mathrm{ml}$. Cell suspensions $(100 \mu \mathrm{l})$ were added to the upper chamber, and the lower chamber was injected with $500 \mu \mathrm{l}$ cell culture medium containing $20 \%$ FBS. Cells were incubated for $24 \mathrm{~h}$ at $37^{\circ} \mathrm{C}$ with $5 \% \mathrm{CO}_{2}$.

The upper chamber was washed with PBS twice and cells under the chamber membrane were fixed with $4 \%$ paraformaldehyde for $5-10 \mathrm{~min}$ at $4^{\circ} \mathrm{C}$, then stained with $1 \%$ crystal violet for $10 \mathrm{~min}$ at room temperature. The stained cells were washed with PBS twice to wipe the staining fluid off of the membrane. Cells were passed through the filter, and cotton tips were used to remove cells remaining on the upper membrane. The invasion of cells was evaluated with an inverted fluorescent microscope (magnification, x200, Olympus). Each group was set triplicate wells.

Semi-quantitative-PCR. Total RNA derived from challenged $\mathrm{FaDu}$ cell lines was isolated using TRIzol ${ }^{\circledR}$ reagent (Invitrogen; Thermo Fisher Scientific, Inc.), according to the manufacturer's protocol. The quality of total RNA was detected by electrophoresis on a $1.2 \%$ agarose gel. The concentration of RNA was measured using a NanoDrop 2000c Spectrophotometer (Thermo Fisher Scientific, Inc.). First strand cDNA was synthesized from total RNA using Fast Reverse Transcriptase (Tiangen Biotech Co., Ltd.), following the manufacturer's instructions. Semi-quantitative PCR was used to detect mRNA expression levels of related genes. The PCR product was visualized by agarose gel containing $0.5 \mathrm{mg} / \mathrm{ml}$ ethidium bromide (EB). And the grey scale value of PCR band was analyzed with ImageJ software v1.8.0 (National Institutes of Health). The primers were designed to amplify specific fragments. The primer sequences of detected genes are presented in Table I.

The amplification was performed in a total volume of $20 \mu 1$, containing $10 \mu \mathrm{l} 2 \mathrm{X}$ Taq PCR Master Mix (Tiangen Biotech Co., Ltd.), $1 \mu \mathrm{l}$ diluted cDNA $(1 \mu \mathrm{g} / \mu \mathrm{l}), 0.5 \mu \mathrm{l}$ each primer $(10 \mu \mathrm{M})$ and $8 \mu \mathrm{l}$ nuclease-free water. Thermocycling conditions for GPR81, TOMM20, PFK-1 and actin genes were as follows: $94^{\circ} \mathrm{C}$ for $3 \mathrm{~min}$ as initial denaturation, 35 cycles of $94^{\circ} \mathrm{C}$ for $30 \mathrm{sec}, 56^{\circ} \mathrm{C}$ for $30 \mathrm{sec}, 72^{\circ} \mathrm{C}$ for $40 \mathrm{sec}$, then $72^{\circ} \mathrm{C}$ 
Table I. Primer sequences.

\begin{tabular}{llrl}
\hline Name & \multicolumn{1}{c}{ Sequence, 5'-3' $^{\prime}$} & Length, nucleotides & Tm, ${ }^{\circ} \mathrm{C}$ \\
\hline GPR81-F1 & TGGCTGCGGACAGGTATT & 18 & 58.8 \\
GPR81-R1 & CCAAACAATCTTGAAGGAGC & 20 & 57.8 \\
TOMM20-F1 & GACCGCAAAAGACGAAGTG & 19 & 57.3 \\
TOMM20-R1 & GGAACACTGGTGGTGGAAG & 19 & 58.2 \\
PFK-1-F1 & TCAGAAGAGGGCAAAGGC & 18 & 58.6 \\
PFK-1-R1 & GCTTGAGCCACCACTGTTCT & 19 & 58.4 \\
MCT1-F1 & TCAAAATAGTCCGATGCC & 18 & 51.0 \\
MCT1-R1 & TGCTGTTTTCCTTCTGCC & 18 & 53.9 \\
Caspase-3-F1 & GAATGACATCTCGGTCTGGT & 20 & 19 \\
Caspase-3-R1 & TGTCTCAATGCCACAGTCC & 18 & 54.1 \\
Bcl-2-F2 & TCCAGGATAACGGAGGCT & 20 & 53.8 \\
Bcl-2-R2 & CACTTGTGGCTCAGATAGGC & 18 & 54.9 \\
$\beta$-actin-F1 & TTGGCAATGAGCGGTTCC & 19 & 55.5 \\
$\beta$-actin-R1 & GAAGGTGGACAGCGAGGC & 58.3 \\
\hline
\end{tabular}

Tm, melting temperature; F, forward; R, reverse; GPR81, G protein-coupled receptor 81; PFK-1, phosphofructokinase; TOMM20, translocase of outer mitochondrial membrane 20; MCT1, monocarboxylase transporter 1 .

extension for $10 \mathrm{~min}$. For caspase-3 and Bcl-2, the same thermocycling conditions were used, except for annealing, which was carried out at $53^{\circ} \mathrm{C}$ for $30 \mathrm{sec}$. The annealing temperature of MCT1 was $51^{\circ} \mathrm{C}$, and the other steps were identical to other genes. All PCR products were held at $16^{\circ} \mathrm{C}$ at the final stage of PCR before detection on an agarose gel.

Western blotting. Cells were lysed with cold RIPA protein lysis buffer supplemented with protease inhibitor (Beijing Solarbio Science \& Technology Co., Ltd.), then placed on a horizontal shaking table for $40 \mathrm{~min}$ at $4^{\circ} \mathrm{C}$. Split samples were centrifuged at $12,000 \mathrm{x}$ g for $20 \mathrm{~min}$ at $4^{\circ} \mathrm{C}$. The supernatant was collected to determine protein density using a BCA Protein Assay kit (Beijing Solarbio Science \& Technology Co., Ltd.). The isolated protein was added to 6X SDS-PAGE loading buffer and boiled for $10 \mathrm{~min}$ at $99^{\circ} \mathrm{C}$. The denatured protein $(\sim 40 \mu \mathrm{g})$ was first separated by SDS-PAGE on pre-cast polyacrylamide gels (12\% separating gel; $5 \%$ stacking gel), then transferred to a wet polyvinylidene fluoride membrane. The transferred membrane was dipped in $5 \%$ bovine serum albumin at $37^{\circ} \mathrm{C}$ for $1 \mathrm{~h}$, then incubated with primary antibodies (GPR81, Abcam, cat. no. ab106942, 1:1,000; PFK-1, Santa Cruz Biotechnology, Inc., cat. no. sc-166722, 1:1,000; TOMM20, Abcam, cat. no. ab186734, 1:1,000; ß-actin, Santa Cruz Biotechnology, Inc., cat. no. sc-47778, 1:2,000) at $4^{\circ} \mathrm{C}$ overnight. Antibody dilutions were made according to the manufacturer's protocol. The membrane was washed with TBS with Tween-20 for 5 min three times. The membrane was then incubated with HRP-conjugated goat anti-rabbit IgG antibody (Abcam, cat. no. ab181662, 1:2,000) at room temperature for $2 \mathrm{~h}$. The blots were visualized using an enhanced chemiluminescence detection kit (Thermo Fisher Scientific, Inc.). Grayscale blots were analyzed with ImageJ v1.8.0 (National Institutes of Health).

Apoptosis detection. FaDu cells $\left(\sim 1 \times 10^{5}\right.$-per well) were seeded in 6-well plates were transfected with shRNA-GPR81 and
shRNA-scramble plasmids. Then, the transfected cells were challenged for $24 \mathrm{~h}$ with cisplatin at the concentration of $5 \mu \mathrm{g} / \mathrm{ml}$. Cells were digested with $0.25 \%$ EDTA-trypsin and washed twice with cold PBS. Apoptosis was assessed in each group according to the annexin V-allophycocyanin/7-aminoat inomycin D apoptosis kit protocol (MultiSciences Biotech Co, Ltd.). The apoptotic cell rate was calculated by the sum of Q2 (apoptosis at late phase or necrotic cells) and Q3 (apoptosis at early stage).

Statistical analysis. SPSS 21.0 statistical software (IBM Corp.) was used to analyze the data, using one-way ANOVA followed by Tukey's multiple comparison test. The differences between two groups were analyzed by Student's t-test. All data are presented as the mean $\pm \mathrm{SD}$. P-values were two-sided and $\mathrm{P}<0.05$ was considered to indicate a statistically significant difference.

\section{Results}

Effect of GPR81 on cell proliferation and invasion. The efficiency of GPR81 gene deletion was detected by semi-quantitative PCR and western blotting in FaDu cells transfected with shRNA-GPR81 and shRNA-scramble plasmid vector (Fig. 1). To examine the effect of GPR81 on cell proliferation and invasion, a CCK-8 assay and a Transwell Matrige ${ }^{\mathrm{TM}}$ assay were performed, respectively.

For the CCK-8 assay, the mean OD450 value in the control, shRNA-scramble and shRNA-GPR81 groups increased to different extents, suggesting a difference in proliferative capacity between theshRNA-GPR81, shRNA-scramble and untreated cell groups. The mean values of OD450 in the control group were $0.386 \pm 0.017,0.788 \pm 0.013,1.045 \pm 0.053$ and $1.354 \pm 0.035$ at time points $0,24,48$ and $72 \mathrm{~h}$, respectively. The mean values of OD450 in the shRNA-scramble group were 

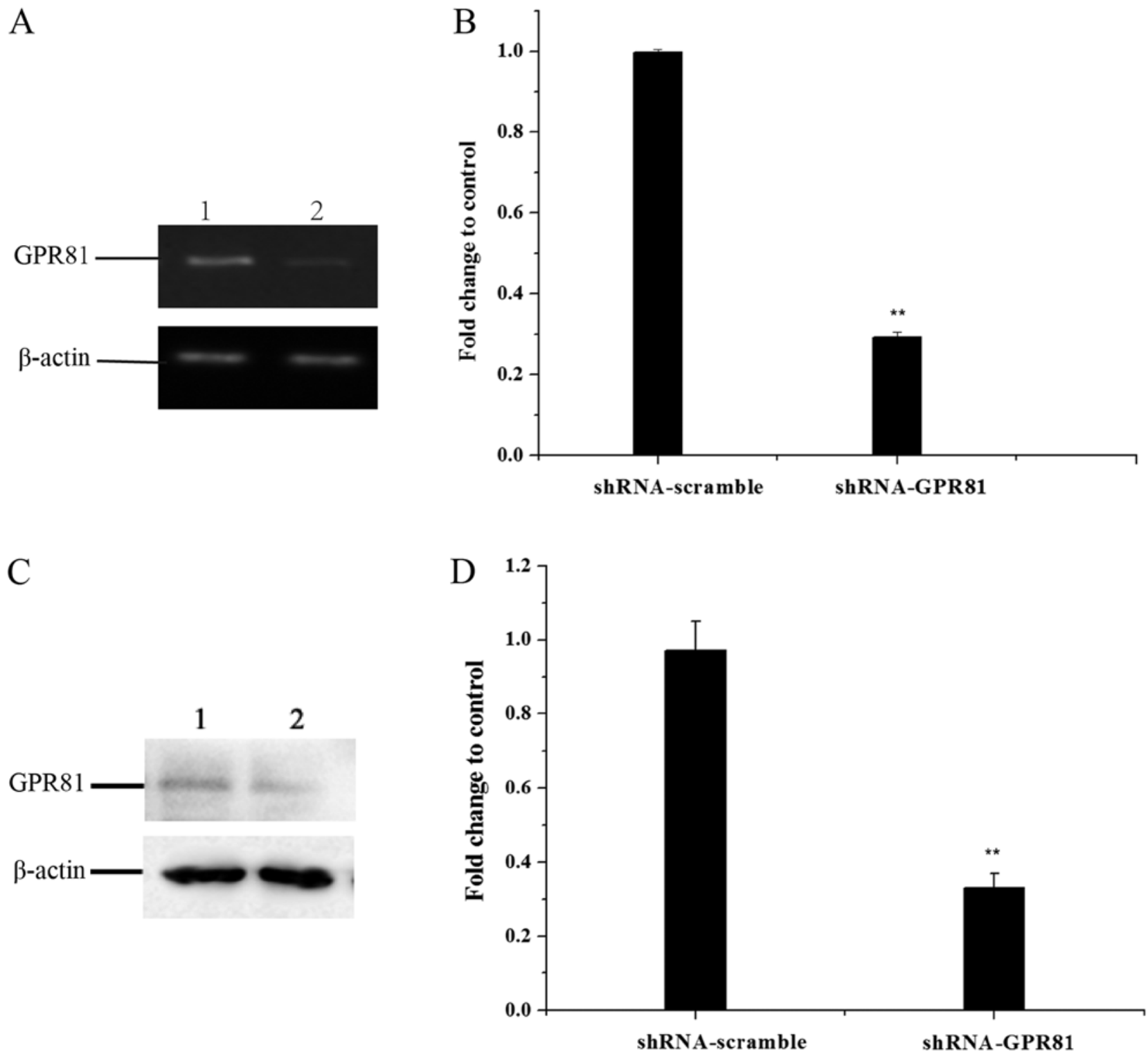

Figure 1. shRNA-GPR81 knockdown decreases GPR81 expression at the mRNA and protein levels in FaDu cells. shRNA-scramble was used as the control. The $\beta$-actin housekeeping gene was used to normalize the protein level. (A) Reverse transcription-PCR detected GPR81 interference efficiency. (B) Quantification of GPR81 interference at the mRNA level. (C) Western blotting detected GPR81 interference efficiency at the protein level. (D) Quantification of GPR81 interference at the protein level. ${ }^{* *} \mathrm{P}<0.01$ vs. control. 1, FaDu cells transfected with shRNA-scramble vector; 2, FaDu cells transfected with shRNA-GPR81 vector; GPR81, G protein-coupled receptor 81; shRNA, short hairpin RNA.

$0.38 \pm 0.012,0.689 \pm 0.065,0.932 \pm 0.051$ and $1.194 \pm 0.08$ at 0,24 , 48 and $72 \mathrm{~h}$, respectively. This was not statistically significant, compared with the control group $(\mathrm{P}>0.05)$. The mean OD450 values in the shRNA-GPR 81 group at $0,24,48$ and $72 \mathrm{~h}$, were $0.367 \pm 0.01,0.451 \pm 0.02,0.634 \pm 0.046$ and $0.838 \pm 0.066$, respectively. At the time points of 48 and $72 \mathrm{~h}$, the OD450 values in shRNA-GPR81 group were significantly lower compared with the control group and shRNA-scramble group (with ${ }^{*} \mathrm{P}<0.05$ vs. control and ${ }^{\#} \mathrm{P}<0.05$ vs. shRNA-scramble), indicating that silencing GPR81 could inhibit the proliferation of FaDu cells (Fig. 2A). These results suggested that GPR81 affected cell proliferation.

In the Transwell Matrigel ${ }^{\mathrm{TM}}$ assay, the mean number of cells passing through the membrane in the control, shRNA-scramble and shRNA-GPR 81 groups were $1.726 \pm 0.098 \times 10^{4}, 1.5677 \pm 0.082 \times 10^{4}$ and $0.514 \pm 0.102 \times 10^{4}$, respectively. The invading cells in the GPR81 silencing group was significantly lower than the shRNA-scramble group (with ${ }^{*} \mathrm{P}<0.05$ vs. control and ${ }^{\#} \mathrm{P}<0.05$ vs. shRNA-scramble; Fig. $2 \mathrm{~B}$ ). These results indicated that silencing GPR81 in FaDu cells inhibited cell invasion.

GPR81 combined with cisplatin affects the expression of genes involved in glycolysis and OXPHOS. RT-semi quantitative PCR and western blotting were used to detect the expression of PFK-1, TOMM20 and MCT1 in FaDu cells at the nucleic acid and protein levels. The influence of cisplatin challenge on PFK-1, TOMM20 and MCT-1 is shown in Fig. S1.

The mRNA expression of PFK-1 in GPR81 knockdown cells combined with cisplatin stimulation slightly decreased, but there was no significant difference compared with untreated cells $(\mathrm{P}>0.05)$. PFK-1 in cells transfected with 
A

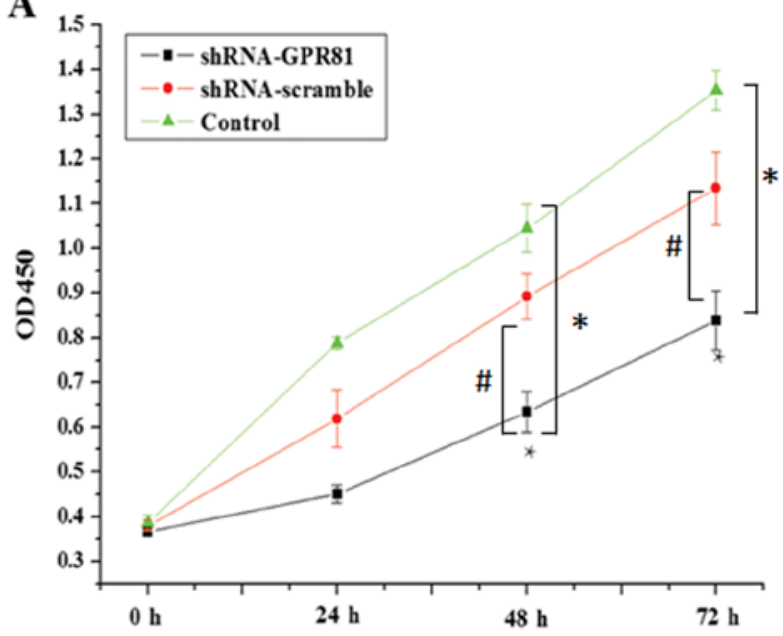

B

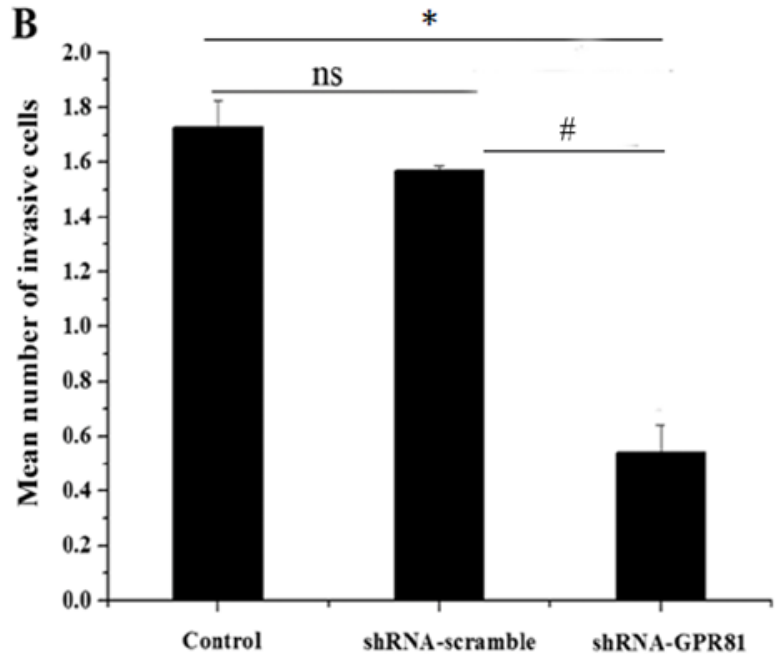

Figure 2. GPR81 knockdown and $5 \mu \mathrm{g} / \mathrm{ml}$ cisplatin treatment inhibit cell proliferation and invasion. (A) Proliferation in shRNA-GPR81, shRNA-scramble and untransfected group. OD450 of the shRNA-GPR81 group was significantly lower than the other groups at 48 and $72 \mathrm{~h}$. ${ }^{*} \mathrm{P}<0.05 \mathrm{vs}$. control; ${ }^{\#} \mathrm{P}<0.05 \mathrm{vs}$. shRNA scramble. (B) Number of invading cells after GPR81 knockdown. Number of invading FaDu cells in the shRNA-GPR81 group was significantly reduced compared with the other groups. ${ }^{*} \mathrm{P}<0.05$ vs. control; ${ }^{*} \mathrm{P}<0.05$ vs. shRNA scramble. OD, optical density; GPR 81 , G protein-coupled receptor 81 ; shRNA, short hairpin RNA; ns, not significant.

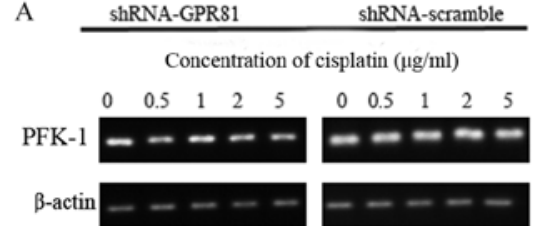

D

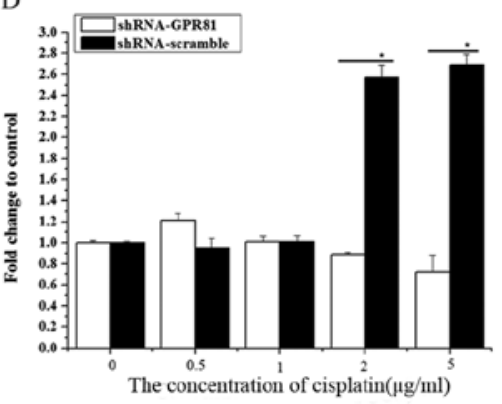

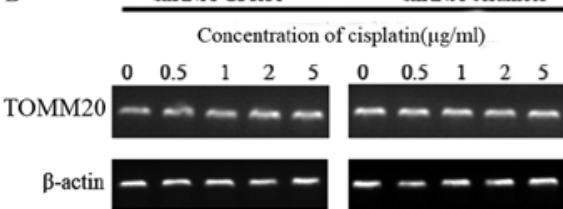

E

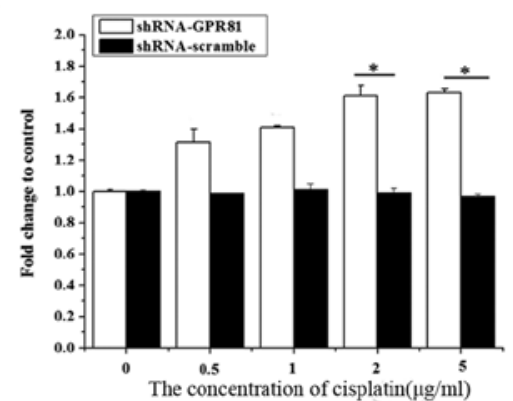

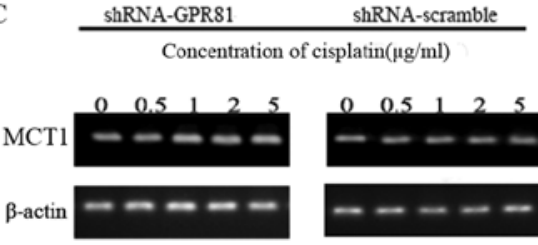

$\mathrm{F}$

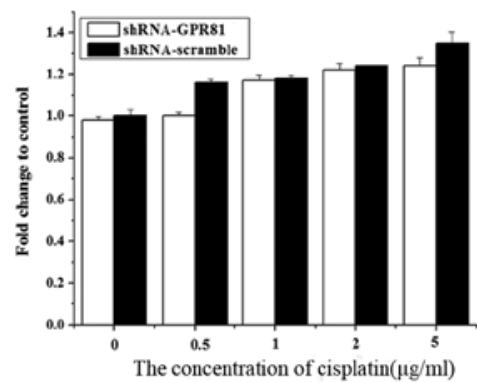

Figure 3. GPR81 silencing combined with cisplatin affects glycolysis and oxidative phosphorylation at the mRNA level. Effects of different concentrations of cisplatin on PFK-1, TOMM20 and MCT1 transcription in FaDu cells after GPR81 silencing. Semi-quantitative PCR was performed to detect mRNA levels of (A) PFK-1, (B) TOMM20 and (C) MCT1. Quantification of (D) PFK-1, (E) TOMM20 and (F) MCT1 mRNA levels. Data are presented as fold change in the mean \pm SD relative to untreated cells. Gene expression was normalized to the housekeeping gene actin. $n=3$ in each group. ${ }^{*} \mathrm{P}<0.05$ vs. the shRNA-scramble group. GPR81, G protein-coupled receptor 81; PFK-1, phosphofructokinase; TOMM20, translocase of outer mitochondrial membrane 20; MCT1, monocarboxylase transporter 1; shRNA, short hairpin RNA.

shRNA-scramble significantly increased rapidly at higher concentrations of cisplatin, which were significantly higher than shRNA-GPR81 cells $(\mathrm{P}<0.05)$. At 2 and $5 \mu \mathrm{g} / \mathrm{ml}$ cisplatin, the difference between two groups was statistically significant $(\mathrm{P}<0.05)$. These results indicated that GPR81 combined with cisplatin might have some influence on the expression of PFK-1 compared with shRNA-scramble group, but no significant effect vs. untransfected cells (Fig. 3A and D).

For the TOMM20 gene, the mRNA expression increased significantly after challenge with cisplatin in GPR81 silenced cells, and peaked at the highest concentration of cisplatin, which was 1.63-fold higher than the control (Fig. 3B and E). The expression of TOMM20 in the shRNA-scramble group increased slightly after being stimulated with cisplatin, with no significant difference compared with untreated cells. However, the expression of TOMM20 increased significantly at 2 and $5 \mu \mathrm{g} / \mathrm{ml}$ cisplatin $(\mathrm{P}<0.05$; Fig. $3 \mathrm{E})$. This suggested that OXPHOS might be affected by GPR81 and cisplatin treatment, through changes in the expression of TOMM20.

The expression of MCT1 gradually increased in both groups stimulated by cisplatin. In addition, there was no significant difference compared with untreated cells, indicating that MCT1 was not affected by GPR81 after challenge with cisplatin (Fig. 3C and F).

At the protein level,PFK-1 expression in GPR81 knockdown cells markedly decreased after cisplatin treatment (lowest value 
A

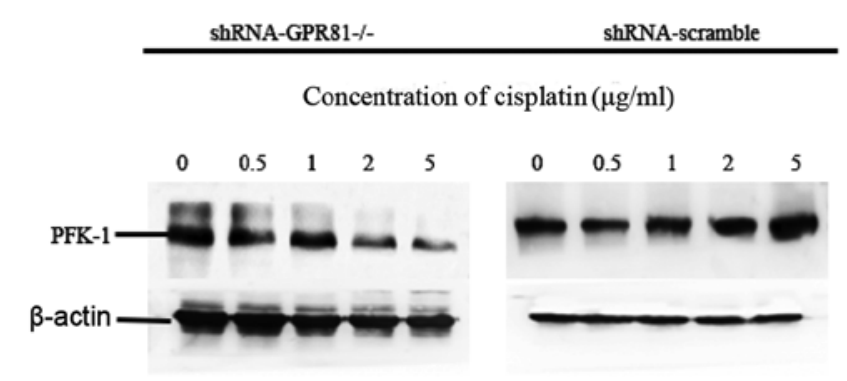

$\mathrm{C}$

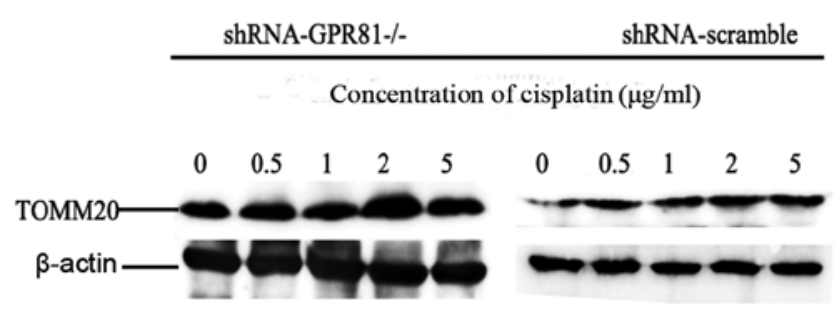

E

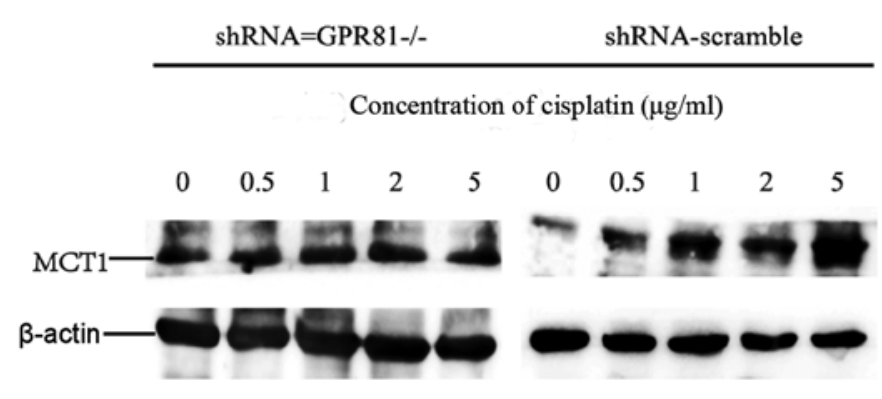

B

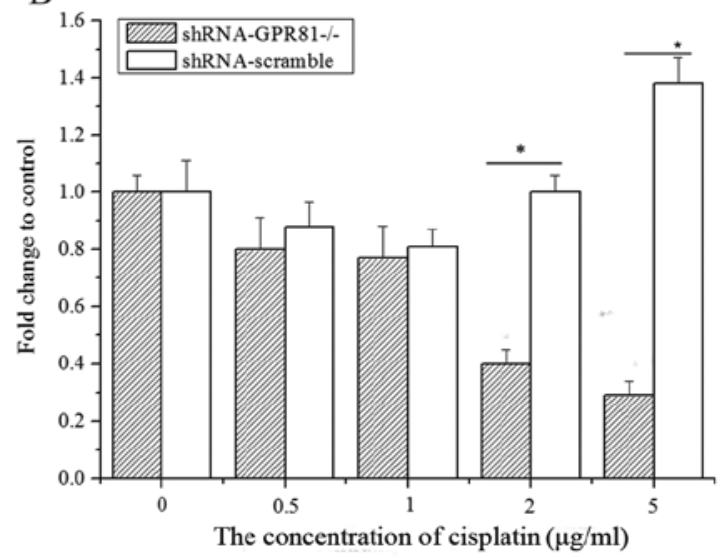

$\mathrm{D}$

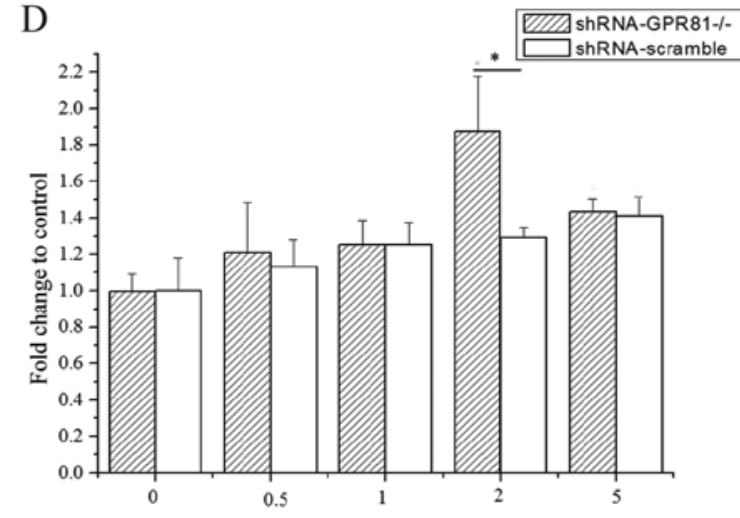

$\mathrm{F}$

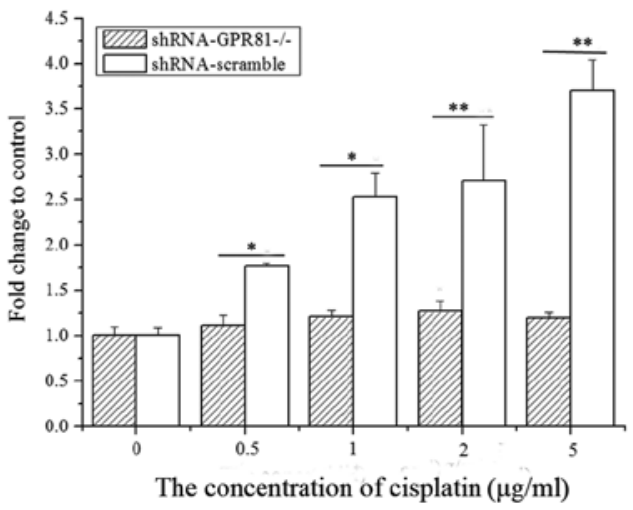

Figure 4. GPR81 silencing combined with cisplatin affects glycolysis and oxidative phosphorylation at the protein level. Effects of different concentrations of cisplatin stimulation on the protein level expression of PFK-1, TOMM20 and MCT1 in FaDu cells after GPR81 silencing. (A and B) Expression of PFK-1 at the protein level. (C and D) Protein expression of TOMM20. (E and F) Expression of MCT1 at the protein level. Protein expression was determined by western blotting and is presented as fold change relative to the untreated group. Protein expression was normalized to the housekeeping gene actin. Data are presented as the mean \pm SD. $n=3$. ${ }^{*} \mathrm{P}<0.05$ and ${ }^{* *} \mathrm{P}<0.01$ vs. the untreated group. GPR81, G protein-coupled receptor 81; PFK-1, phosphofructokinase; TOMM20, translocase of outer mitochondrial membrane 20; MCT1, monocarboxylase transporter 1; shRNA, short hairpin RNA.

$\sim 0.29$-fold of control at the highest concentration of cisplatin). However, the expression of PFK-1 in the shRNA-scramble group was increased after cisplatin challenge (Fig. 4A and B). The expression of PFK-1 with 2 and $5 \mu \mathrm{g} / \mathrm{ml}$ cisplatin incubation in shRNA-GPR81 and shRNA-scramble groups exhibited a significant difference $(\mathrm{P}<0.05)$. These results indicated that GPR81 affected the levels of PFK-1, which in turn might inhibit glycolysis following challenge with cisplatin.

For TOMM20, the total expression trend of the protein level was similar to that of the mRNA level, with a slight upregulation in both shRNA-GPR81 and shRNA-scramble groups. TOMM20 protein expression was the highest at a concentration of $2 \mu \mathrm{g} / \mathrm{ml}$ cisplatin. The expression of TOMM 20 in shRNA-GPR81 was significantly higher compared with the shRNA-scramble group $(\mathrm{P}<0.05)$ after challenge with $22 \mu \mathrm{g} / \mathrm{ml}$ cisplatin. These results suggested that cisplatin stimulation might enhance the process of OXPHOS in cancer cells (Fig. 4C and D).

The expression of MCT1 in two groups manifested upregulation following cisplatin incubation. However, the expression 

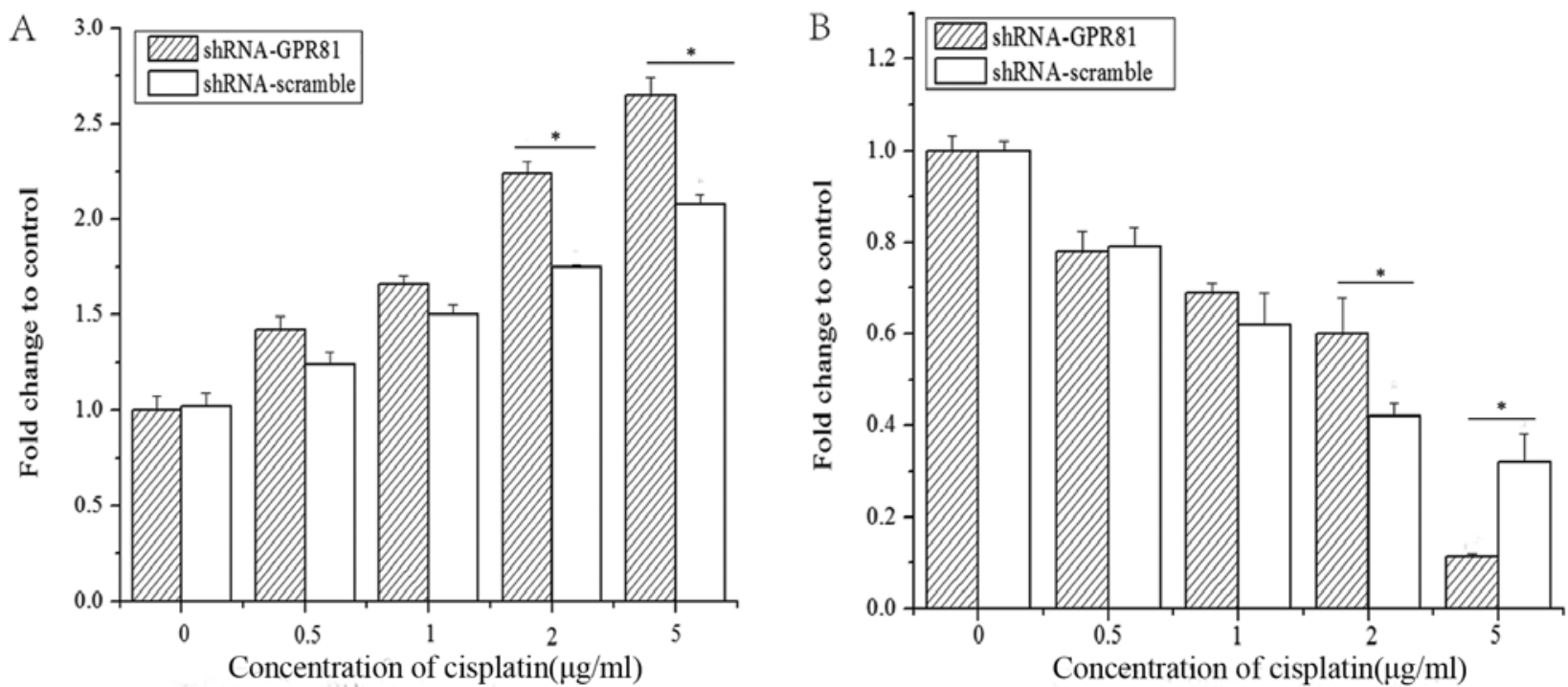

Figure 5. mRNA expression of caspase-3 and Bcl-2 in cisplatin-stimulated FaDu cells transfected with shRNA-GPR81 or shRNA-scramble. (A) Caspase-3 transcription. (B) Bcl-2 transcription. Data are presented as fold change in the mean $\pm \mathrm{SD}$, relative to untreated cells. Gene expression was normalized to the housekeeping gene actin. $n=3$. ${ }^{*} \mathrm{P}<0.05$ vs. the shRNA-scramble group. GPR81, G protein-coupled receptor 81 ; shRNA, short hairpin RNA.

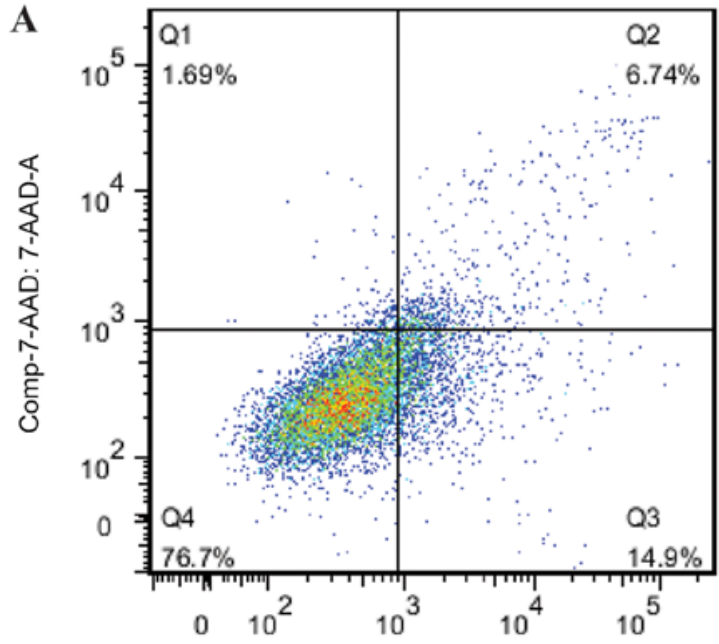

Comp-APC-A: Annexin V APC-A

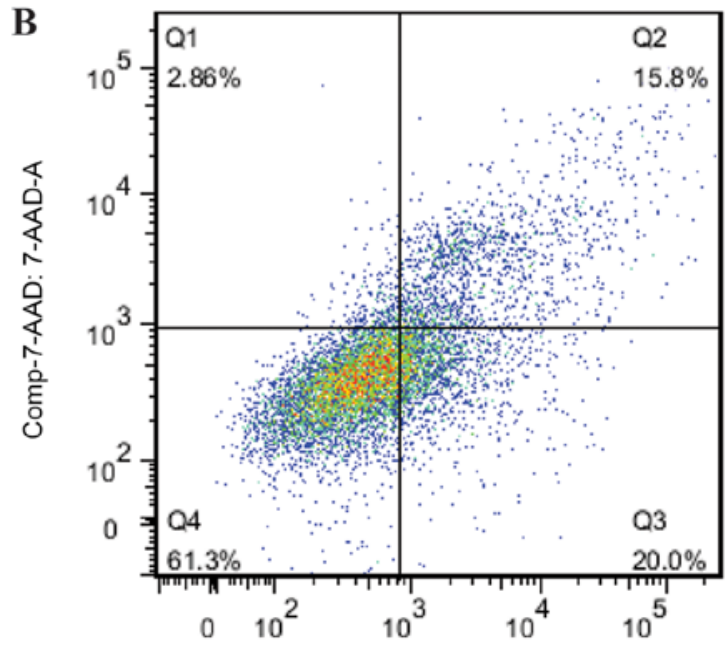

Comp-APC-A: Annexin V APC-A

C

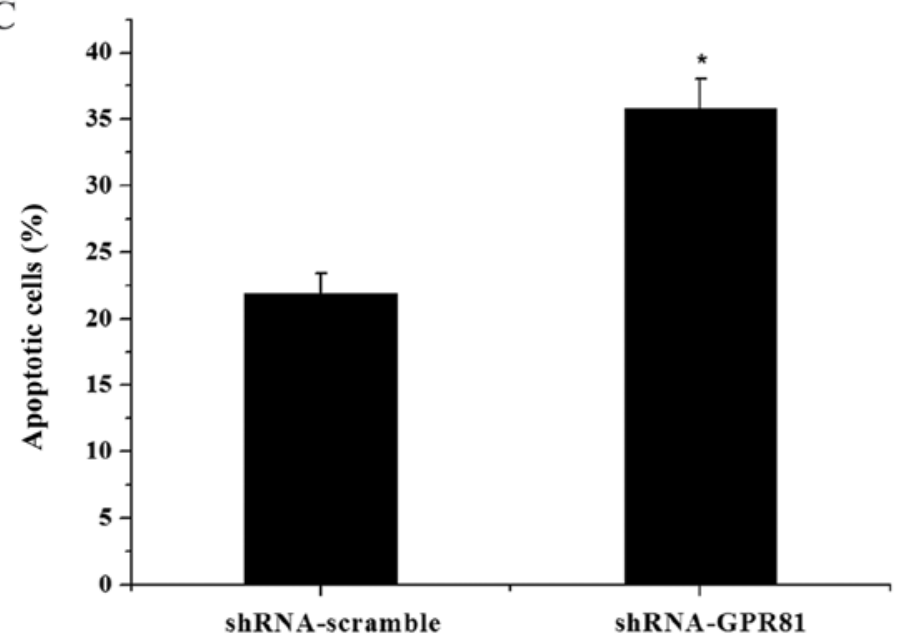

Figure 6. GPR81 knockdown combined with cisplatin increases apoptosis of FaDu cells. Flow cytometry was used to analyze apoptosis in shRNA-GPR81 and shRNA-scramble transfected FaDu cells. (A) Apoptosis in shRNA-scramble group. (B) GPR81 knockdown triggers cell apoptosis. (C) Quantification of apoptosis. $\mathrm{n}=3$. ${ }^{*} \mathrm{P}<0.05$ vs. the shRNA-scramble group. GPR81, G protein-coupled receptor 81; shRNA, short hairpin RNA; 7-AAD, 7'aminoactinomycin D; APC, allophycocyanin. 
of MCT1 in shRNA-scramble group was significantly higher than that in shRNA-GPR81 group after stimulation by 0.5 and $1 \mu \mathrm{g} / \mathrm{ml}$ cisplatin $(\mathrm{P}<0.05)$, and significant differences were found after stimulation by 2 and $5 \mu \mathrm{g} / \mathrm{ml}$ cisplatin $(\mathrm{P}<0.01$; Fig. 4E and F).

GPR81 silencing combined with cisplatin promotes apoptosis in FaDu cells. The mRNA expression profile of caspase-3 and Bcl-2 in GPR81-silenced cells after cisplatin stimulation was also assessed (Fig. 5). In both the shRNA-GPR81 silencing group and shRNA-scramble group, the expression of caspase-3 was gradually elevated, to a peak at $5 \mu \mathrm{g} / \mathrm{ml}$ of cisplatin. Following incubation with 2 and $5 \mu \mathrm{g} / \mathrm{ml}$ cisplatin, the expression of caspase- 3 in shRNA-GPR 81 and shRNA-scramble groups showed a significant difference $(\mathrm{P}<0.05)$. For the $\mathrm{Bcl}-2$ gene, mRNA expression decreased in shRNA-GPR81 and shRNA-scramble groups, to $\sim 0.1$ and 0.3 -fold of the untreated cells group. A significant difference in the expression of Bcl-2 was observed in the shRNA-GPR81 and shRNA-scramble groups at 2 and $5 \mu \mathrm{g} / \mathrm{ml}$ cisplatin $(\mathrm{P}<0.05)$. These results suggested that GPR81 affected apoptosis following cisplatin treatment. Flow cytometry analyses showed that the apoptotic cell rate of the shRNA-GPR81 group was higher than that of the shRNA-scramble group $(\mathrm{P}<0.05$; Fig. 6$)$. The results indicated that GPR81 combined with cisplatin enhanced apoptosis in cancer cells.

\section{Discussion}

The present study demonstrated the influence of GPR81 on energy metabolism and apoptosis in hypopharyngeal carcinoma in vitro, using an shRNA interference technique combined with the chemotherapeutic agent cisplatin. The results of the present study suggested that GPR81 gene knockdown inhibited cell proliferation and invasion. Moreover, GPR81 silencing combined with cisplatin affects the expression of PFK-1, TOMM20 and MCT1, which are involved in energy metabolism and lactate transport. Furthermore, cisplatin treatment increased apoptosis in GPR81-silenced cells, as indicated by altered levels ofcaspase-3. Altogether, the findings of the present study suggested that GPR81 knockdown might increase the efficacy of chemotherapy agents, such as cisplatin, enhance apoptosis and influence the pathways related to energy metabolism.

As a crucial lactate receptor, GPR81 directly affects metabolites involved in tumor cell survival and energy metabolism, altering the uptake of glucose and modulating the normal process in mammalian organisms by activating various receptors (28). A similar observation regarding the role of GPR81 was made in breast cancer and pancreatic carcinoma cell lines $(29,30)$. These previous studies indicated that the absence of GPR81 in breast cancer cells and pancreatic carcinoma cell lines markedly decreased cell proliferation and invasion, leading to cell death in vitro.

GPR81 also functions as a vital regulator of cell survival by inhibiting apoptosis and promoting cell survival $(19,18)$. In the present study, it was hypothesized that the inhibition of GPR81 on cell proliferation would depend on apoptosis. Cisplatin is a conventional chemotherapeutic reagent used in the treatment of various tumor types (30), and blocks malignant cell growth through various mechanisms, such as DNA damage, cell cycle deregulation, apoptosis and autophagy (31-33). Our previous study demonstrated that cisplatin treatment enhanced apoptosis in FaDu cells (34). GPR81 knockdown in FaDu cells led to an increase in apoptosis in the presence of cisplatin. In the present study, the proportion of apoptotic cells was also significantly increased in shRNA-GPR81 FaDu cells. In addition, the expression of caspase-3 increased, while the expression of $\mathrm{Bcl}-2$ decreased in the presence of cisplatin. A similar conclusion regardingGPR81 was also previously suggested in HeLa cells, demonstrating that silencing GPR81 accelerated apoptosis in another in vitro system (16). Therefore, GPR81 regulates survival and apoptosis in hypopharyngeal carcinoma cells.

The coexistence of glycolysis with the generation of lactate and mitochondrial OXPHOS is a hallmark in carcinoma cells $(35,36)$. Lactate is a vital bridge in energy metabolism. The lack of the lactate receptor GPR81 would affect the content of lactate and ultimately influence biological metabolism. The present study examined whether the absence of GPR81 and cisplatin stimulation would have an impact on glycolysis and OXPHOS by detecting key markers at the mRNA and protein levels. It was demonstrated that shRNA-GPR81 combined with cisplatin decreased PFK-1 mRNA and protein levels, but increased TOMM20 and MCT1 mRNA and protein levels to varying degrees. PFK-1 is essential for glycolysis, as it catalyzes rate-limiting steps of fructose 6-phosphate and ATP conversion into fructose 1,6-bisphosphate and ADP (37). A previous study identified that cisplatin treatment decreased glycolysis by inhibiting PFK-1 expression (38). In the present study, the importance of GPR81 in triggering cisplatin efficacy and weakening the glycolysis pathway was demonstrated in HSCC. Thus, reduced expression of PFK- 1 could contribute to the alteration of lactate, allowing tumor cells to alter glycolysis to survive in a complex micro-environment.

TOMM20, a pivotal translocase located in the outer mitochondrial membrane, has been reported as susceptible to cisplatin. In addition, the protein level of TOMM20 was increased when incubated with cisplatin in cholangiocarcinoma cells (39). However, the relationship between GPR81 and TOMM20 is still ambiguous. In our previous study, cisplatin induced a slight downregulation of TOMM20 in FaDu cells (34). In the present study, the elevated mRNA and protein levels of TOMM20 could be caused by changes in GPR81levels in the presence of cisplatin.

MCT1 is involved in cellular uptake of lactate. The expression of MCT1 is induced by lactate, and accumulation of lactate also increases the expression of MCT1 (40). The absence of GPR81 affects lactate binding, leading to lactate accumulation in cells. Moreover, expression of MCT1 was correlated with cisplatin-resistance in epithelial ovarian tumors (41). In epithelial ovarian tumors, the expression of MCT1 was enhanced after treatment with cisplatin. Hu et al (42) demonstrated that both GPR81 and MCT1 were highly expressed in squamous carcinoma; however, the expression of GPR81 and MCT1 had no association in squamous carcinoma. In the present study, MCT1 showed a marginal increase in GPR81-silenced cells and after cisplatin treatment. Thus, depletion of GPR81could change the concentration of lactate, which would indirectly influence the process of lactate uptake. Finally, changes in the 
expression of MCT1 were due to a combined effect of GPR81 and cisplatin.

In summary, the present study suggested a critical role for GPR81 in hypopharyngeal carcinoma following cisplatin treatment. Moreover, the present study provided an insight into the mechanism underlying the cellular metabolism and resistance to cisplatin through PFK-1, TOMM20 and MCT1. Notably, GPR81 was crucial for cell proliferation and invasion, which may account for the changes in energy metabolism alteration seen in hypopharyngeal cancer.

\section{Acknowledgements}

Not applicable.

\section{Funding}

The research was supported by Excellent Talents Plan Foundation of Hebei province (grant no. 361004) and Hebei province Natural Science Foundation (grant no. H2016206535).

\section{Availability of data and materials}

The datasets used and/or analyzed during the current study are available from the corresponding author on reasonable request.

\section{Authors' contributions}

JW performed the of cell culture and cell challenge. OX performed the of semi-quantitative PCR and analyzed the data. JD designed the sequence of primers used in the present study. XJ performed the RNA extraction and protein lysis. QJ performed cell transfection, cell proliferation, invasion, western blotting and apoptosis assays, and was a major contributor in writing the manuscript. XR analyzed the western blotting data and revised the manuscript. CS designed the present study and revised the manuscript. All authors read and approved the final manuscript.

\section{Ethics approval and consent to participate}

Not applicable.

\section{Patient consent for publication}

Not applicable.

\section{Competing interests}

The authors declare that they have no competing interests.

\section{References}

1. Cohen N, Fedewa S and Chen AY: Epidemiology and demographics of the head and neck cancer population. Oral Maxillofac Surg Clin North Am 30: 381-395, 2018.

2. Bradley PJ: Epidemiologyz of hypopharyngeal cancer. Adv Otorhinolaryngol 83: 1-14, 2019.

3. Znaor A, Brennan P, Gajalakshmi V, Mathew A, Shanta V, Varghese $C$ and Boffetta P: Independent and combined effects of tobacco smoking, chewing and alcohol drinking on the risk of oral, pharyngeal and esophageal cancers in Indian men. Int J Cancer 105: 681-686, 2003.
4. Mehanna H, Beech T, Nicholson T, El-Hariry I, McConkey C, Paleri V and Roberts S: Prevalence of human papillomavirus in oropharyngeal and nonoropharyngeal head and neck cancer-systematic review and meta-analysis of trends by time and region. Head Neck 35: 747-755, 2013.

5. Newman JR, Connolly TM, Illing EA, Kilgore ML, Locher J and Carroll WR: Survival trends in hypopharyngeal cancer: A population-based review. Laryngoscope 125: 624-629, 2015.

6. American Cancer Society: Laryngeal and hypopharyngeal cancer. http: //www.cancer.org/cancer/laryngealandhypopharyngealcancer/index. Accessed January 4, 2015.

7. Hanahan D and Weinberg RA: Hallmarks of cancer: The next generation. Cell 144: 646-674, 2011.

8. Moreno-Sánchez R, Rodríguez-Enríquez S, Marín-Hernández A and Saavedra E: Energy metabolism in tumor cells. FEBS J 274 1393-1418, 2007.

9. Fantin VR, St-Pierre J and Leder P: Attenuation of LDH-A expression uncovers a link between glycolysis, mitochondrial physiology, and tumor maintenance. Cancer Cell 9: 425-434, 2006.

10. Díaz-Muñiz I, Banavara DS, Budinich MF, Rankin SA, Dudley EG and Steele JL: Lactobacillus casei metabolic potential to utilize citrate as an energy source in ripening cheese: A bioinformatics approach. J Appl Microbiol 101: 872-882, 2006.

11. Brodsky AN, Odenwelder DC and Harcum SW: High extracellular lactate causes reductive carboxylation in breast tissue cell lines grown under normoxic conditions. PLoS One 14: e0213419, 2019.

12. Grasmann G, Smolle E, Olschewski H and Leithner $\mathrm{K}$ : Gluconeogenesis in cancer cells-Repurposing of a starvation-induced metabolic pathway? Biochim Biophys Acta Rev Cancer 1872: 24-36, 2019.

13. Liu C, Wu J, Zhu J, Kuei C, Yu J, Shelton J, Sutton SW, Li X, Yun SJ, Mirzadegan T, et al: Lactate inhibits lipolysis in fat cells through activation of an orphan G-protein-coupled receptor, GPR81. J Biol Chem 284: 2811-2822, 2009.

14. Rooney K and Trayhurn P: Lactate and the GPR81 receptor in metabolic regulation: Implications for adipose tissue function and fatty acid utilisation by muscle during exercise. Br J Nutr 106: 1310-1316, 2011.

15. Morland C, Lauritzen KH, Puchades M, Holm-Hansen S, Andersson K, Gjedde A, Attramadal H, Storm-Mathisen J and Bergersen LH: The lactate receptor, G-protein-coupled receptor 81/hydroxycarboxylic acid receptor 1: Expression and action in brain. J Neurosci Res 93: 1045-1055, 2015.

16. Wagner W, Kania KD, Blauz A and Ciszewski WM: The lactate receptor (HCAR1/GPR81) contributes to doxorubicin chemoresistance via ABCB1 transporter up-regulation in human cervical cancer HeLa cells. J Physiol Pharmacol 68: 555-564, 2017.

17. Feng J, Yang H, Zhang Y, Wei H, Zhu Z, Zhu B, Yang M, Cao W, Wang $\mathrm{L}$ and $\mathrm{Wu} \mathrm{Z}$ : Tumor cell-derived lactate induces TAZ-dependent upregulation of PD-L1 through GPR81 in human lung cancer cells. Oncogene 36: 5829-5839, 2017.

18. Roland CL, Arumugam T, Deng T, Liu SH, Philip B, Gomez S, Burns WR, Ramachandran V, Wang H, Cruz-Monserrate $\mathrm{Z}$ and Logsdon CD: Cell surface lactate receptor GPR81 is crucial for cancer cell survival. Cancer Res 74: 5301-5310, 2014.

19. Caslin HL, Abebayehu D, Abdul Qayum A, Haque TT, Taruselli MT, Paez PA, Pondicherry N, Barnstein BO, Hoeferlin LA, Chalfant CE and Ryan JJ: Lactic acid inhibits lipopolysaccharide-induced mast cell function by limiting glycolysis and ATP availability. J Immunol 203: 453-464, 2019.

20. Ideno M, Kobayashi M, Sasaki S, Futagi Y, Narumi K, Furugen A and Iseki K: Involvement of monocarboxylate transporter 1 (SLC16A1) in the uptake of l-lactate in human astrocytes. Life Sci 192: 110-114, 2018.

21. Curry JM, Tuluc M, Whitaker-Menezes D, Ames JA, Anantharaman A, Butera A, Leiby B, Cognetti DM, Sotgia F, Lisanti MP, et al: Cancer metabolism, stemness and tumor recurrence: MCT1 and MCT4 are functional biomarkers of metabolic symbiosis in head and neck cancer. Cell Cycle 12: 1371-1384, 2013.

22. Yuan Y, Guo-Qing $\mathrm{P}$, Yan T, Hong-Lin Y, Gong-Hua $\mathrm{H}$ and Cai-Gao Z: A study of PKM2, PFK-1, and ANT1 expressions in cervical biopsy tissues in China. Med Oncol 29: 2904-2910, 2012.

23. Gooptu M, Whitaker-Menezes D, Sprandio J, Domingo-Vidal M, Lin Z, Uppal G, Gong J, Fratamico R, Leiby B, Dulau-Florea A, et al: Mitochondrial and glycolytic metabolic compartmentalization in diffuse large B-cell lymphoma. Semin Oncol 44: 204-217, 2017. 
24. Sotgia F, Whitaker-Menezes D, Martinez-Outschoorn UE, Flomenberg N, Birbe RC, Witkiewicz AK, Howell A, Philp NJ, Pestell RG and Lisanti MP: Mitochondrial metabolism in cancer metastasis: Visualizing tumor cell mitochondria and the 'reverse Warburg effect' in positive lymph node tissue. Cell Cycle 11: $1445-1454,2012$.

25. Zhao Z, Han F, He Y, Yang S, Hua L, Wu J and Zhan W: Stromal-epithelial metabolic coupling in gastric cancer: Stromal MCT4 and mitochondrial TOMM20 as poor prognostic factors. Eur J Surg Oncol 40: 1361-1368, 2014.

26. Park SH, Lee AR, Choi K, Joung S, Yoon JB and Kim S: TOMM20 as a potential therapeutic target of colorectal cancer. BMB Rep 52: 712-717, 2019.

27. Duan K, Liu ZJ, Hu SQ, Huo HY, Xu ZR, Ruan JF, Sun Y, Dai LP, Yan CB, Xiong W, et al: Lactic acid induces lactate transport and glycolysis/OXPHOS interconversion in glioblastoma. Biochem Biophys Res Commun 503: 888-894, 2018.

28. Brown TP and Ganapathy V: Lactate/GPR81 signaling and proton motive force in cancer: Role in angiogenesis, immune escape, nutrition, and Warburg phenomenon. Pharmacol Ther 206 $107451,2020$.

29. Lee YJ, Shin KJ, Park SA, Park KS, Park S, Heo K, Seo YK, Noh DY, Ryu SH and Suh PG: G-protein-coupled receptor 81 promotes a malignant phenotype in breast cancer through angiogenic factor secretion. Oncotarget 7: 70898-70911, 2016.

30. Rancoule C, Guy JB, Vallard A, Ben Mrad M, Rehailia A and Magné N: 50th anniversary of cisplatin. Bull Cancer 104: 167-176, 2017.

31. Zhang K, Zhang B, Bai Y and Dai L: E2F1 promotes cancer cell sensitivity to cisplatin by regulating the cellular DNA damage response through miR-26b in esophageal squamous cell carcinoma. J Cancer 11: 301-310, 2020.

32. Shi S, Tan P, Yan B, Gao R, Zhao J, Wang J, Guo J, Li N and Ma Z: ER stress and autophagy are involved in the apoptosis induced by cisplatin in human lung cancer cells. Oncol Rep 35: 2606-2614, 2016

33. Zheng AW, Chen YQ, Fang J, Zhang YL and Jia DD: Xiaoaiping combined with cisplatin can inhibit proliferation and invasion and induce cell cycle arrest and apoptosis in human ovarian cancer cell lines. Biomed Pharmacother 89: 1172-1177, 2017.
34. Xu O, Jia QJ, Wang JX and Shan C: A preliminary study on the function of GPR81 and TOMM20 in hypopharyngeal carcinoma. Chin J Otorhinolaryngol Skull Base Surg 25: 498-503, 2019.

35. Ashton TM, McKenna WG, Kunz-Schughart LA and Higgins GS: Oxidative phosphorylation as an emerging target in cancer therapy. Clin Cancer Res 24: 2482-2490, 2018.

36. Bottoni $\mathrm{P}$ and Scatena R: Mitochondrial metabolism in cancer. A tangled topic. Which role for proteomics? Adv Exp Med Biol 1158: 1-16, 2019.

37. Mor I, Cheung EC and Vousden KH: Control of glycolysis through regulation of PFK-1: Old friends and recent additions. Cold Spring Harb Symp Quant Biol 76: 211-216, 2011.

38. Marín-Hernández A, Gallardo-Pérez JC, López-Ramírez SY, García-García JD, Rodríguez-Zavala JS, Ruiz-Ramírez L, Gracia-Mora I, Zentella-Dehesa A, Sosa-Garrocho M, Macías-Silva M, et al: Casiopeina II-gly and bromo-pyruvate inhibition of tumor hexokinase, glycolysis, and oxidative phosphorylation. Arch Toxicol 86: 753-766, 2012

39. Fan Z, Yu H, Cui N, Kong X, Liu X, Chang Y, Wu Y, Sun L and Wang G: ABT737 enhances cholangiocarcinoma sensitivity to cisplatin through regulation of mitochondrial dynamics. Exp Cell Res 335: 68-81, 2015.

40. Lottes RG, Newton DA, Spyropoulos DD and Baatz JE: Lactate as substrate for mitochondrial respiration in alveolar epithelial type II cells. Am J Physiol Lung Cell Mol Physiol 308: L953-L961, 2015.

41. Yan C, Yang F, Zhou C, Chen X,Han X, Liu X, Ma H and Zheng W: MCT1 promotes the cisplatin-resistance by antagonizing Fas in epithelial ovarian cancer. Int J Clin Exp Pathol 8: 2710-2718, 2015.

42. Hu Y and Zeng F: Expressions of GPR81, MCT1 and MCT4 in squamous carcinoma and their clinical significance. Zhong Nan Da Xue Xue Bao Yi Xue Ban 43: 950-956, 2018 (In Chinese).

This work is licensed under a Creative Commons Attribution-NonCommercial-NoDerivatives 4.0 International (CC BY-NC-ND 4.0) License. 